\title{
Massive Purulent Pericardial Effusion Secondary to Knee Infection
}

\author{
Srinath Damodaran ${ }^{1}$, Komal Gandhi ${ }^{2}$, Krishna P Gourav ${ }^{3}$, Rupesh Kumar ${ }^{4}$
}

\begin{abstract}
Perioperative echocardiography is a useful guide in the management of hemodynamically unstable patients. We, hereby, describe a case of massive pericardial effusion with interesting images on echocardiography.

Keywords: Pericardial effusion, Pericardiectomy, Staphylococcus aureus, Transesophageal echocardiography.

Journal of Perioperative Echocardiography (2019): 10.5005/jp-journals-10034-1097
\end{abstract}

\section{Case Description}

A 3-year-old child had history of trivial trauma to left knee 3 months back. Subsequently, he developed high-grade fever and swelling of left knee. Septic knee arthritis was diagnosed and managed with incision and drainage along with intravenous antibiotics. The child was discharged after 1 day of hospital stay. Three months later, the child was referred to our institute with grade III breathlessness, abdominal distension, and bilateral pitting pedal edema. On hospital admission, he found to be febrile with heart rate of $150 /$ minute, blood pressure of $65 / 35 \mathrm{~mm} \mathrm{Hg}$, and room air saturation $\mathrm{SpO}_{2}$ of $92 \%$. Examination revealed engorged jugular veins with muffled heart sounds. Chest X-ray showed a globular-shaped heart. Child underwent bedside transthoracic echocardiography (TTE) which revealed a massive pericardial effusion ( $4 \mathrm{~cm}$ ) (owl's eye appearance on TTE in massive pericardial effusion) in the parasternal shortaxis view (Fig. 1A, see Supplementary data online, Video S1).

In view of hemodynamic instability and massive pericardial effusion, the patient was planned for emergency pericardiectomy. Inside the operating room, after attaching standard monitoring, a femoral arterial cannulation was done under mild sedation. Anesthesia was induced with graded doses of injection ketamine and injection fentanyl $(25 \mu \mathrm{g})$. Intermittent doses of injection phenylephrine was used while induction to prevent hypotension. The transesophageal echocardiography (TEE) after tracheal intubation reconfirmed the preoperative diagnosis of massive pericardial effusion without any diastolic collapse (Figs 1B and C, see Supplementary data online, Videos S2 and S3). The inferior vena cava was dilated with no case pericardium, purulent contents were drained out, and pericardiectomy was performed. Post-procedure TEE showed mild right ventricular (RV) and left ventricular (LV) dysfunction with mild mitral regurgitation (MR) (Figs $1 \mathrm{G}$ to $\mathrm{H}$, see Supplementary data online, Videos S4 to S6). The trachea was extubated after 12 hours. The culture of pericardial fluid had growth of vancomycin-sensitive Staphylococcus aureus.

Collapse of right-sided cardiac chamber is a common sign of cardiac tamponade. ${ }^{1}$ Moreover, duration of atrial collapse longer than one third of the cardiac cycle has been described as an
${ }^{1-3}$ Department of Anesthesia and Intensive Care, Postgraduate Institute of Medical Education and Research, Chandigarh, India

${ }^{4}$ Department of Cardiovascular and Thoracic Surgery, Postgraduate Institute of Medical Education and Research, Chandigarh, India

Corresponding Author: Krishna P Gourav, Department of Anesthesia and Intensive Care, Postgraduate Institute of Medical Education and Research, Chandigarh, India, Phone: +91 8194952345, e-mail: kpgmbbs777@gmail.com

How to cite this article: Damodaran S, Gandhi K, Gourav KP, et al. Massive Purulent Pericardial Effusion Secondary to Knee Infection. J Perioper Echocardiogr 2019;7(1):24-25.

Source of support: Nil

Conflict of interest: None

almost $100 \%$ sensitive and specific sign of cardiac tamponade. ${ }^{2}$ Furthermore, the absence of collapse of any cardiac chamber has a $90 \%$ negative predictive value. ${ }^{3}$ In our case, there was no diastolic collapse of the right atrium or the right ventricle throughout the cardiac cycle. A gradual increase in pericardial collection over 3 months might be the reason for the insidious onset of signs and symptoms. There was a mild RV and LV dysfunctions with mild MR in the postoperative period. This was possibly due to pericardial decompression syndrome. ${ }^{4}$ However, the patient was hemodynamically stable after drainage of pericardial effusion.

\section{Conclusion}

The purulent pericardial effusion secondary to communityacquired, Staphylococcus aureus is a potentially lethal infection and rarely described in the pediatric population. Perioperative echocardiography plays a vital role in the anesthetic management of a case with massive pericardial effusion.

\section{Author Contributions}

Srinath Damodaran prepared the manuscript. Komal Gandhi and Krishna P Gourav managed the case and prepared the manuscript. Rupesh Kumar managed the case.

() The Author(s). 20190pen Access This article is distributed under the terms of the Creative Commons Attribution 4.0 International License (https://creativecommons. org/licenses/by-nc/4.0/), which permits unrestricted use, distribution, and non-commercial reproduction in any medium, provided you give appropriate credit to the original author(s) and the source, provide a link to the Creative Commons license, and indicate if changes were made. The Creative Commons Public Domain Dedication waiver (http://creativecommons.org/publicdomain/zero/1.0/) applies to the data made available in this article, unless otherwise stated. 

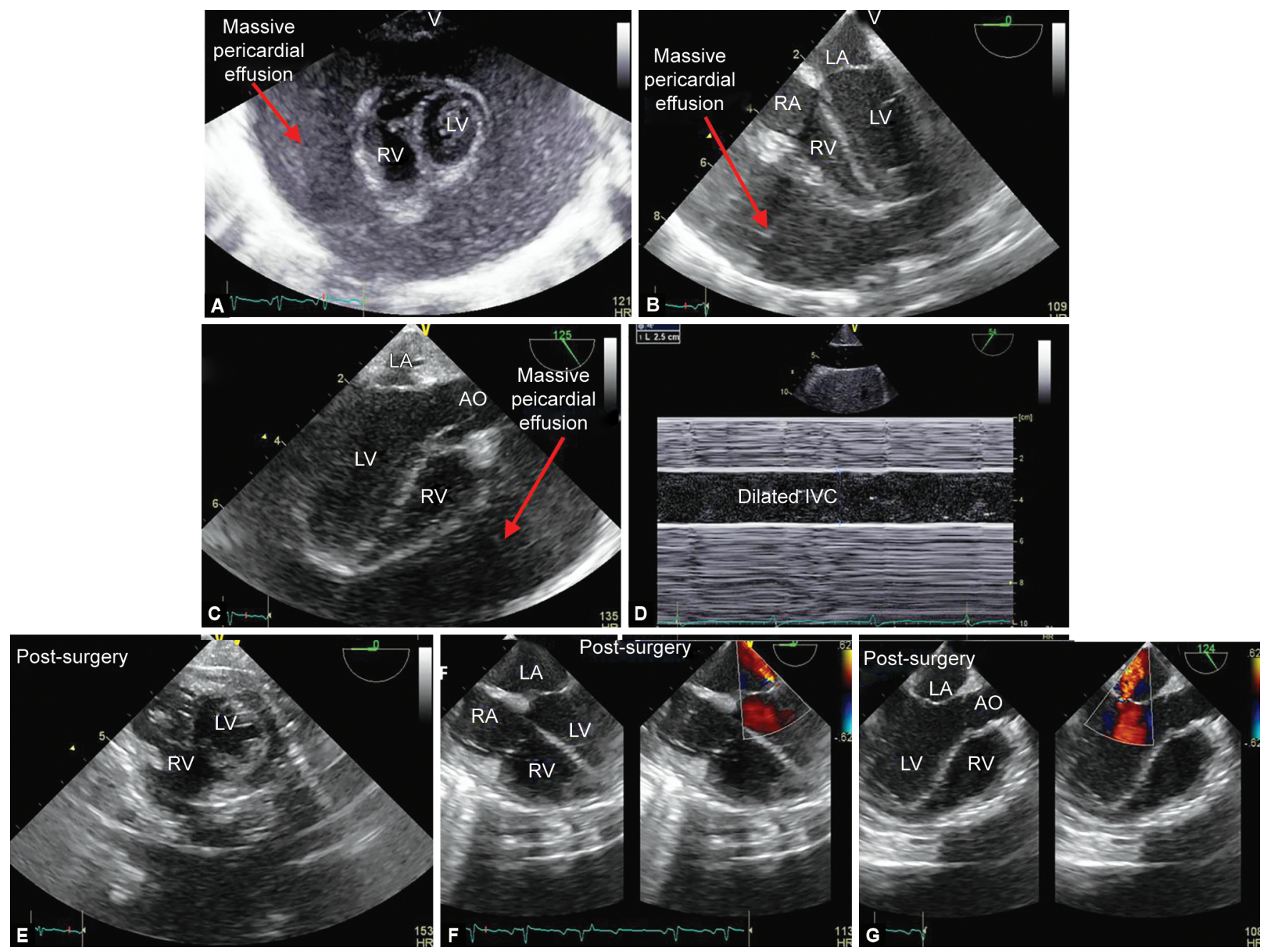

Figs 1 A to G: (A) A transthoracic echocardiographic image showing a massive pericardial effusion in the parasternal shortaxis view (LV, left ventricle; $\mathrm{RV}$, right ventricle); (B) A transesophageal echocardiographic image showing a massive pericardial effusion in the midesophageal four-chamber view (LA, left atrium; LV, left ventricle; RA, right atrium; RV, right ventricle); (C) A transesophageal echocardiographic image showing a massive pericardial effusion in the midesophageal aortic long-axis view (AO, ascending aorta; LA, left atrium; LV, left ventricle; RV, right ventricle); (D) A transesophageal echocardiographic image showing a dilated inferior vena cava (IVC); (E) A transesophageal echocardiographic image showing no pericardial effusion in the transgastric LV midpapillary view after surgical intervention (LV, left ventricle; RV, right ventricle); (F) A transesophageal echocardiographic image with color comparision showing no pericardial effusion, with mild mitral regurgitation in midesophageal four-chamber view after surgical intervention (LA, left atrium; LV, left ventricle; RA, right atrium; RV, right ventricle); (G) A transesophageal echocardiographic image with color comparision showing no pericardial effusion, with mild mitral regurgitation in the midesophageal aortic longaxis view after surgical intervention ( $\mathrm{AO}$, ascending aorta; LA, left atrium; LV, left ventricle; RV, right ventricle)

\section{References}

1. Singh S, Wann LS, Klopfenstein HS, et al. Usefulness of right ventricular diastolic collapse in diagnosing cardiac tamponade and comparison to pulsus paradoxus. Am J Cardiol 1986;57(8):652-656. DOI: 10.1016/0002-9149(86)90853-2.

2. Gillam LD, Guyer DE, Gibson TC, et al. Hydrodynamic compression of the right atrium: a new echocardiographic sign of cardiac tamponade. Circulation 1983;68(2):294-301. DOI: 10.1161/01.cir.68.2.294.
3. Merce J, SagristaSauleda J, PermanyerMiralda G, et al. Correlation between clinical and Doppler echocardiographic findings in patients with moderate and large pericardial effusion: implications for the diagnosis of cardiac tamponade. Am Heart J 1999;138(4 Pt 1):759-764. DOI: 10.1016/s0002-8703(99)70193-6.

4. Ayoub C, Chang M, Kritharides L. A case report of ventricular dysfunction post pericardiocentesis: stress cardiomyopathy or pericardial decompression syndrome? Cardiovasc Ultrasound 2015;13(1):32. DOI: 10.1186/s12947-015-0026-3.

Video S1: A transthoracic echocardiographic image showing a massive pericardial effusion in parasternal shortaxis view

Video S2: A transesophageal echocardiographic image showing a massive pericardial effusion in midesophageal four-chamber view

Video S3: A transesophageal echocardiographic image showing a massive pericardial effusion in midesophageal aortic long axis view

Video S4: A transesophageal echocardiographic image showing massive pericardial effusion in transgastric left ventricular midpapillary view

Video S5: A transesophageal echocardiographic image showing no pericardial effusion, with mild mitral regurgitation in midesophageal four-chamber view after surgical intervention

Video S6: A transesophageal echocardiographic image showing no pericardial effusion, with mild mitral regurgitation in midesophageal aortic long axis view after surgical intervention 